DOI: $10.15593 / 24111678 / 2016.04 .01$

УДК 551.509.32

\author{
Т.Г. Анодина ${ }^{1}$, И.Ш. Магеррамов ${ }^{2}$ \\ ${ }^{1}$ Межгосударственный авиационный комитет, Москва, Россия \\ ${ }^{2}$ Национальное аэрокосмическое агентство, \\ Баку, Азербайджанская Республика

\section{ИССЛЕДОВАНИЕ ОПТИМАЛЬНОГО СООТНОШЕНИЯ АЭРОЗОЛЯ ДЛЯ ДОСТИЖЕНИЯ НАИЛУЧШЕЙ ВИДИМОСТИ В АЭРОПОРТАХ} \\ КОНЦЕНТРАЦИЙ СОСТАВЛЯЮЩИХ АТМОСФЕРНОГО
}

\begin{abstract}
Известно, что самолеты эмитируют в атмосферу такие токсичные газы, как диоксид углерода, окиси озона и серы, а также аэрозоли. Авиация оказывает прямое воздействие на климат, состав атмосферы в зоне полетов и качество воздуха в окрестностях аэропортов. Аэрозоли рассеивают и частично поглощают солнечное излучение, а также радиацию, излучаемую земными объектами, вследствие чего происходит ухудшение видимости. Одной из базовых формул для вычисления видимости является формула Кошмидера. Однако она является слишком обобщенной и не учитывает такие специфические физические процессы, как увлажнение аэрозоля, многокомпонентность аэрозоля, динамические изменения структурного состава аэрозоля и др. В данной статье исследуется вопрос об обеспечении требуемой видимости при разных соотношениях концентрации составляющих двухсоставного увлажненного аэрозоля. Многочисленние исследования корреляционной связи между среднесуточной видимостью и среднесуточной величиной $\mathrm{PM}_{10}$ показывают наличие гиперболической зависимости между видимостью и концентрацией $\mathrm{PM}_{10}$. В статье показано, что аналогичный результат может быть получен аналитически, путем анализа результатов экспериментально-модельных исследований. Представлено также, что при взаимно инверсном характере изменений обьемных концентраций составных частей двухсоставного аэрозоля в течение серии проводимых экспериментов при наложении ограничения на интегральную величину видимости в атмосфере, загрязненной двухсоставным аэрозолем, интегральная величина экстинкции достигает минимальной величины. Указанная закономерность может иметь некоторое прикладное значение в плане обеспечения требуемой видимости в зонах аэропортов, в том числе на взлетных полосах.
\end{abstract}

Ключевые слова: видимость, оптимизация, аэропорты, аэрозоль, увлажнение, концентрация.

\author{
T.G. Anodina ${ }^{1}$, I.Sh. Magerramov ${ }^{2}$ \\ ${ }^{1}$ Intergovernmental Aviation Committee, Moscow, Russian Federation \\ ${ }^{2}$ National Aerospace Agency, Baku, Azerbaijan Republic
}

\title{
RESEARCH OF OPTIMUM RATIO OF CONCENTRATIONS OF COMPONENTS OF ATMOSPHERIC AEROSOL TO REACH THE MAXIMUM VISIBILITY IN AIRPORTS
}

It is well-known that airplanes emit to atmosphere such toxical gases as carbon dioxide, ozone oxide, sulphur oxide and aerosol. The aviation directly impacts on climate and atmospheric components in flight zone and on air quality in vicinity of airports. Aerosol scatter and partially absorbs the solar radiation and the radiation of objects on the Earth which leads to decrease of visibility. One of basic formulaes used 
for calculation of visibility is the formula of Koshmider. Despite its universal designation this formula is too generalized because doesn't takes into account such specific physical processes as humidification of aerosol, multicomponent structure and dynamic changes of structure of aerosol. In the article the question on provision of the required visibility upon different ratios of concentrations of components of humidified aerosol is studied. The multiple researches of correlation between average daily visibility and average daily $\mathrm{PM}_{10}$ confirm the presence of hyperbolic dependence between visibility and concentration of $\mathrm{PM}_{10}$. In the article it is shown that the similar result can be derived analytically by way analysis of results of experimental-model researches. It is also shown that upon mutually inverse character of changes of volume concentrations of components of aerosol during the series of held experiments and upon the limitation of integral value of visibility in the atmosphere polluted by multicomponent aerosol the integrated value of extinction reaches the minimum value. Such a regularity may has some applied importance for provision the required visibility in airport zones, including in taking-off and landing routes.

Keywords: visibility, optimization, airports, aerosol, humidification, concentration.

Хорошо известно, что авиация оказывает прямое воздействие на климат, состав атмосферы в зоне полетов и качество воздуха в окрестностях аэропортов, поскольку самолеты эмитируют в атмосферу такие токсичные газы, как диоксид углерода, окиси озона и серы, а также аэрозоли [1-8]. Аэрозоли рассеивают и частично поглощают солнечное излучение, а также радиацию, излучаемую земными объектами. Диаметр аэрозольных частиц, эмитируемых самолетами, находится в переделах от 100 нм до 3 мкм [1]. Согласно работе [2] исследования, проведенные в окрестностях аэропорта г. Лос-Анжелеса, показали, что при наличии ветров на расстоянии 8-10 км концентрация аэрозоля повышается в 4-5 раз, а на расстоянии 16 км - в 2 раза. Указанные факты подтверждают актуальность проведения исследований по изучению аэрозолей на видимость в зоне аэропортов. Согласно работам [3, 8-12] видимость определяется в качестве такой максимальной горизонтальной дистанции, на которой объекты видны невооруженным глазом. Дистанция видимости (VIS) определяется по формуле Кошмидера [12]

$$
k_{\mathrm{ext}}=\frac{3,912}{\mathrm{VIS}} .
$$

При этом $k_{\text {ext }}$ определяется как

$$
k_{\text {ext }}=k_{\text {ext PM }}+k_{\text {ext } F}+k_{\text {ext air }},
$$

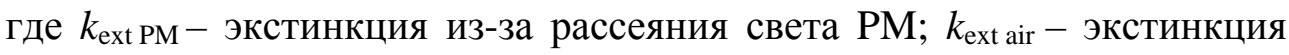
из-за рассеяния молекулами воздуха; $k_{\mathrm{ext} F}-$ экстинкция, возникающая из-за других причин.

Вместе с тем формула Кошмидера является слишком обобщенной и не учитывает такие специфические физические процессы, как увлажнение аэрозоля, многокомпонентность аэрозоля, динамические изменения структурного состава аэрозоля и др. В настоящей статье ис- 
следуется вопрос об обеспечении требуемой видимости при разных соотношениях концентрации составляющих двухсоставного увлажненного аэрозоля.

Согласно работам [4, 13-15] существует следующая зависимость между объемной концентрацией аэрозоля и относительной влажностью:

$$
k_{\mathrm{ex}}=k \cdot V^{a}(1-\mathrm{RH})^{-b \cdot \mathrm{RH}},
$$

где $k_{\mathrm{ex}}$ - коэффициент экстинкции, ммоль; $k$ - среднее значение, $k=\frac{k_{\text {ех }}}{V}$, ммоль $\left(\text { мкм }^{3} \cdot \mathrm{cm}^{-3}\right)^{-1} ; V-$ объемная концентрация, мкм ${ }^{3} / \mathrm{cm}^{3}$; $\mathrm{RH}$ - относительная влажность; $a=0,944 ; b=0,475 ; k=9,08$ [4].

Рассмотрим следующую модельную оптимизационную задачу. Допустим, в атмосфере имеется двухсоставной аэрозоль, коэффициенты экстинкции которого определяются как

$$
\begin{gathered}
k_{\mathrm{ex} 1}=k_{1} \cdot V_{1}^{a_{1}}(1-\mathrm{RH})^{-b_{1} \cdot \mathrm{RH},}, \\
k_{\mathrm{ex} 2}=k_{2} \cdot V_{2}^{a_{2}}(1-\mathrm{RH})^{-b_{2} \cdot \mathrm{RH},},
\end{gathered}
$$

где $k_{1}, k_{2}, a_{1}, b_{1}, a_{2}, b_{2}=$ const [ 4 ].

Введем на рассмотрение искомую функцию $V_{2}=\varphi\left(V_{1}\right)$. С учетом выражений (4) и (5) определим коэффициент экстинкции для двухсоставного аэрозоля:

$$
k_{\text {дв. ех }}=k_{1} V_{1}^{a_{1}}(1-\mathrm{RH})^{-b_{1} \cdot \mathrm{RH}}+k_{2} \varphi\left(V_{1}\right)^{a_{2}}(1-\mathrm{RH})^{-b_{2} \cdot \mathrm{RH}} .
$$

Составим функционал оптимизации в смысле нахождения оптимальной функции $V_{2 o p t}=\varphi\left(V_{1}\right)_{\text {opt }}$ при которой интегральный функционал

$$
k_{\text {дв. ех ин. }}=\int_{0}^{V_{1 \max }}\left[k_{1} \cdot V_{1}^{a_{1}}(1-\mathrm{RH})^{-b_{1} \cdot \mathrm{RH}}+k_{2} \varphi\left(V_{1}\right)^{a_{2}} \cdot(1-\mathrm{RH})^{-b_{2} \cdot \mathrm{RH}}\right] d V_{1}
$$

достиг бы экстремальной величины при соблюдении некоторого ограничительного условия.

Для формирования ограничительного условия проанализируем результаты экспериментальных исследований, проведенных в работах $[5,6]$. Как отмечается в работе [5], исследования корреляционной связи между среднесуточной видимостью и среднесуточной величиной $\mathrm{PM}_{10}$ в течение 2003-2007 гг. в г. Пекине показали наличие гиперболической зависимости между видимостью и концентрацией $\mathrm{PM}_{10}$ (рис. 1). 


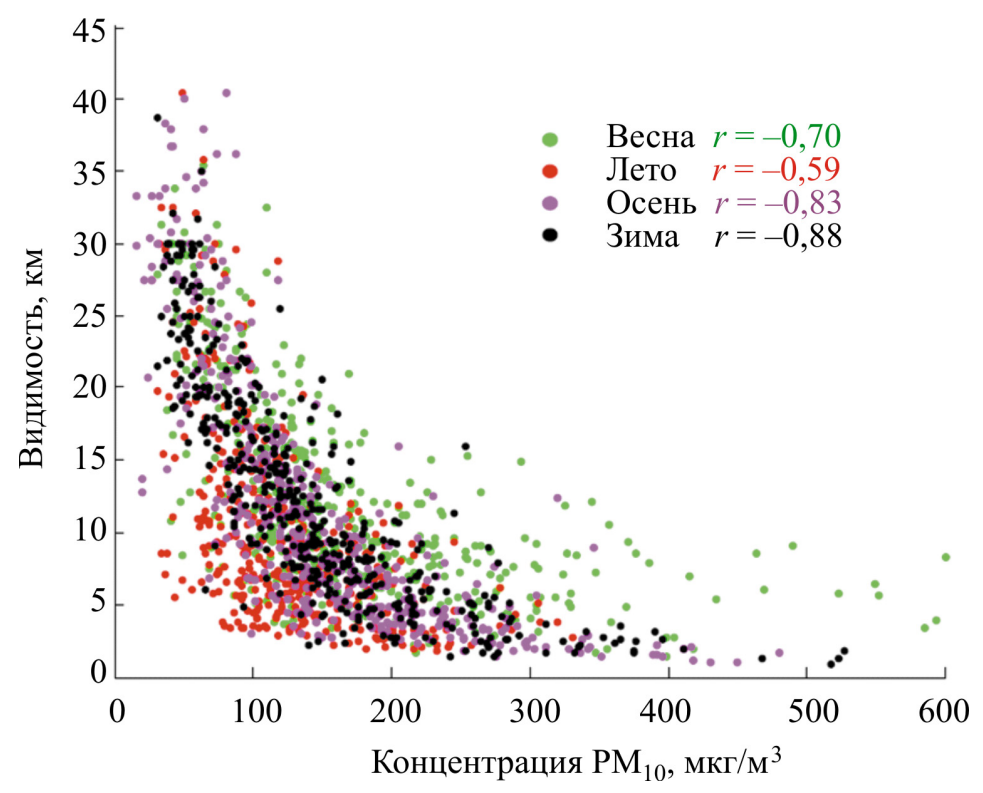

Рис. 1. Гиперболическая зависимость между видимостью и концентрацией $\mathrm{PM}_{10}$, полученная в работе [5]

Отметим, что аналогичный результат может быть получен путем анализа результатов экспериментально-модельных исследований, представленных в работе [6]. В данной статье изложены результаты исследования изменения видимости в результате увеличения концентрации некоторых видов аэрозоля, включая крупнодисперсный минеральный аэрозоль, нерастворимый аэрозоль, минеральный аэрозоль аккумуляционной моды, водорастворимый аэрозоль при $80 \%$ относительной влажности, водорастворимый аэрозоль при $70 \%$ относительной влажности, водорастворимый аэрозоль при $50 \%$ относительной влажности, сажу. Соответствующие графики приведены на рис. 2. Если проанализировать кривые 4-6, то они с точностью $\pm 5 \%$ аппроксимируются функцией

$$
\mathrm{VIS}=A \cdot \exp [-(\log N) \cdot \mathrm{RH}]
$$

Далее воспользуемся результатами работ [7, 13], согласно которым коэффициент гигроскопического роста может быть аппроксимирован экспоненциальной функцией зависимости от RH, т.е.

$$
f=e^{d \cdot \mathrm{RH}},
$$

где $d$ - коэффициент пропорциональности. 


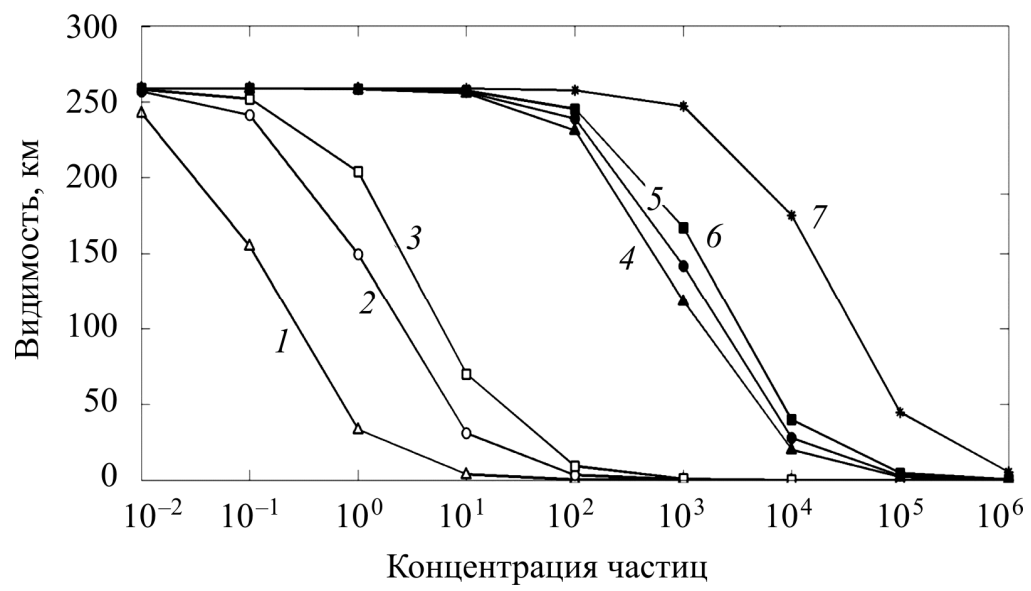

Рис. 2. Кривые зависимости видимости от концентрации различных видов аэрозоля, полученные экспериментально-модельным путем

Из выражений (8) и (9) имеем:

$$
\mathrm{VIS}=A \cdot \exp \left[-\frac{(\log N) \cdot \log f}{d}\right]
$$

Из формулы (10) при $\log f=d$ получаем

$$
\mathrm{VIS}=A / N \text {, }
$$

что совпадает с результатом, представленным в работе [5].

С учетом вышеизложенного для окончательного формирования оптимизационной задачи определения оптимального соотношения двух разных типов аэрозолей в смысле получения экстремальной величины интегрированного значения экстинкции оптической радиации по результатам серии экспериментов сформируем следующее ограничительное условие:

$$
\int_{0}^{V_{1} \max } \frac{B}{V_{1}+\varphi\left(V_{1}\right)} d V_{1}=C
$$

где $B, C=$ const.

Отметим, что физическим смыслом ограничения (12) является обеспечение постоянства интегрированной по всей серии экспериментов видимости.

С учетом выражений (7) и (12) составим полный функционал безусловной вариационной оптимизации: 


$$
\begin{gathered}
k_{\text {дв. ех ин }}=\int_{0}^{V_{1} \max }\left[k_{1} V_{1}^{a_{1}}(1-\mathrm{RH})^{-b_{1} \cdot \mathrm{RH}}+k_{2} \varphi\left(V_{1}\right)^{a_{1}}(1-\mathrm{RH})^{-b_{2} \cdot \mathrm{RH}}\right] d V_{1}+ \\
+\lambda \int_{0}^{V_{1} \max } \frac{B \cdot d V_{1}}{V_{1}+\varphi\left(V_{1}\right)}
\end{gathered}
$$

где $\lambda$ - множитель Лагранжа.

Согласно методу Эйлера оптимальная функция $\varphi\left(V_{1}\right)_{\text {opt }}$ должна удовлетворить следующему условию:

$$
\frac{d\left[k_{1} V_{1}^{a_{1}}(1-\mathrm{RH})^{-b_{1} \cdot \mathrm{RH}}+k_{2} \varphi\left(V_{1}\right)^{a_{1}} \cdot(1-\mathrm{RH})^{-b_{2} \cdot \mathrm{RH}}+\frac{\lambda \cdot B}{V_{1}+\varphi\left(V_{1}\right)}\right]}{d \varphi\left(V_{1}\right)}=0 .
$$

Из выражения (14) получим

$$
k_{2} \cdot a_{1} \cdot \varphi\left(V_{1}\right)^{a_{1}-1}(1-\mathrm{RH})^{-b_{2} \cdot \mathrm{RH}}-\frac{\lambda \cdot B}{\left[V_{1}+\varphi\left(V_{1}\right)\right]^{2}}=0 .
$$

Из формул (12) и (15) нетрудно получить следующее выражение для вычисления множителя Лагранжа:

$$
\lambda=\lambda_{0}=\left[\frac{1}{C} \int_{0}^{V_{1} \max } \sqrt{k_{2} a_{2}} f\left(V_{1}\right)^{a_{2}-1}(1-\mathrm{RH})^{-b_{2} \cdot \mathrm{RH}} \cdot B d V_{1}\right]^{2} .
$$

С учетом формулы (16) решение оптимизационной задачи получено в виде

$$
f\left(V_{1}\right)=\sqrt{\frac{\lambda_{0} \cdot B}{k_{2} a_{2}(1-\mathrm{RH})^{-b_{2} \cdot \mathrm{RH}}}}-V_{1} .
$$

Для проверки характера экстремума функционала (13) достаточно вычислить знак следующего выражения и убедиться, что он положителен, т.е. решение (17) обеспечивает минимальную величину $k_{\text {дв. ех ин: }}$ :

$$
F=\frac{d^{2}\left[k_{1} V_{1}^{a_{1}}(1-\mathrm{RH})^{-b_{1} \cdot \mathrm{RH}}+k_{2} \varphi\left(V_{1}\right)^{a_{1}} \cdot(1-\mathrm{RH})^{-b_{2} \cdot \mathrm{RH}}+\frac{\lambda \cdot B}{V_{1}+\varphi\left(V_{1}\right)}\right]}{d \varphi\left(V_{1}\right)^{2}} .
$$


Следовательно, показано, что при взаимно инверсном характере изменений $V_{1}$ и $V_{2}$ в течение серии проводимых экспериментов при наложении ограничения на интегральную величину видимости в атмосфере, загрязненной двухсоставным аэрозолем, интегральная величина экстинкции достигает минимальной величины. Указанная закономерность может иметь некоторое прикладное значение в плане обеспечения требуемой видимости в зонах аэропортов, в том числе на взлетных полосах.

\section{Список литературы}

1. Impact of al ternative jet fuels on aircraft-induced aerosols / C. R ojo, X. Vancassel, P. M irabel, J.-L. Ponche, F. Garnier // Fuel. - 2015. Vol. 144. - P. 335-341.

2. Emissions from an international airport increase number concentrations 4-fold at $10 \mathrm{~km}$ downwind / N. Hudda, T. Gould, K. Hartin, T.V. Larson, S.A. Fruin // Environmental Science \& Technology. - 2014. - № 48. P. 6628-6635.

3. Mukherjee A., Toohey D.W. A study of aerosol properties based on observations of particulate matter from the U.S. Embassy in Beijing, China // Earth's Future. - 2016. - Vol. 4, iss. 8. - P. 381-395. DOl: $10.1002 / 2016$ EF 000367

4. A parameterization of low visibilities for hazy days / J . Chen [et al.] // A tmospheric Chemistry and Physics. - 2012. - Vol. 12. - P. 4935-4950.

5. Zhang Q.H., Zhang J.P. Xue H.W. The challenge of improving visibility in Beijing // Atmospheric Chemistry and Physics. - 2010.Vol. 10. - P. 7821-7827.

6. Singh A., Dey S. Influence of aerosol composition on visibility in megacity Delhi // A tmospheric Environment. - 2012. - V ol. 62. - P. 367-373.

7. A new experimental approach to study the hydroscopic and optical properties of aerosol: application to ammonium sulfate particles / C. Denjean, P. Formenti, B. Picquet-V arrault, Y . K atrib, E. Pangui, P. Zapf, J.F. Doussin // A tmospheric M easurement Techniques. - 2014. - V ol. 7. - P. 183-197.

8. M ali R.R., V ashistha R.D. V isibility measurement technique and its application in aviation services at international airports in India. - URL: https: $\quad$ www.wmo.int/pages/prog/www/IM OPpublications/IOM -82-TECO2005/Posters/P1(08)_India_2_M ali.pdf (дата обращения: 01.09.2016).

9. Okey K., N w ofor Ph.D. Seasonal levels of meteorological visibility at port-harcourt Nigeria and possible links to aerosol loading and humidifi- 
cation // The Pacific J ournal of Science and Technology. - 2010. - Vol. 11, № 2. - URL: http: //www.akamaiuniversity.us/PJ ST.htm (дата обращения: 01.09.2016).

10. Evaluation of aerosol distribution and optical depth in the geophysical fluid dynamics laboratory coupled model CM 2.1 for present climate / P. Ginoux, L.W. Horowitz, V. Ramaswamy, I.V. Geogdzhayev, B.N. Holben, G. Stenchikov, X. Tie // J ournal of Geophysical Research. - 2006. - V ol. 111, D22210. - P. 1-21. DOI: 10.1029/2005J D 006707

11. Impact of relative humidity and water soluble constituents of $P M_{2,5}$ on visibility impairment in B eijing, China / J. Chen, Sh. Qiu, J. Sjang, M.F.O. Wilfrid, X. Liu, H. Tian, J. Boman // A erosol and Air Quality Research. - 2014. - Vol. 14. - P. 260-268.

12. The impacts of aerosol loading, composition and water uptake on aerosol extinction variability in the Baltimore - Washington, D.C. region / A.J. B eyersdorf, L.D. Ziemba, G. Chen, C.A. Corr, J.H. Crawford, G.S. Diskin, R.H. M oore, K.L. Thornhill, E.L. W instead, B.E. A nderson // A tmospheric Chemistry and Physics. - 2016. - Vol. 16. - P. 1003-1015.

13. Petters M.D., K reidenwais S.M . A single parameter representation of hydroscopic growth and cloud condensation nucleus activity // A tmospheric Chemistry and Physics. - 2007. - V ol. 7. - P. 1961-1971.

14. M easuring air pollutants in an international Romania airport with point and open path instruments / I. Ionel, D. Nicolae, F. Popescu, C. Talianu, J. Belegante, G. A postol // Romanian Journal of Physics. - 2011. - Vol. 56, № 3-4. - P. 507-519.

15. Daley P.S., Naugle D.F. M easurements and analysis of airport emissions // J ournal of Air Pollution Control A ssociation. - 1979. - Vol. 29. P. 113-116.

\section{References}

1. R ojo C., V ancassel X., M irabel P., Ponche J.-L ., Garnier F. Impact of alternative jet fuels on aircraft-induced aerosols. Fuel, 2015, vol. 144, pp. 335-341.

2. Hudda N., Gould T., Hartin K., Larson T.V ., Fruin S.A. Emissions from an international airport increase number concentrations 4-fold at $10 \mathrm{~km}$ downwind. Environmental Science \& Technology, 2014, no. 48, pp. 6628-6635.

3. Mukherjee A., Toohey D.W. A study of aerosol properties based on observations of particulate matter from the U.S. Embassy in Beijing, China. Earth's Future, 2016, vol. 4, iss. 8, pp. 381-395. DOI: 10.1002/2016EF000367 
4. Chen J. [et al.]. A parameterization of low visibilities for hazy days. Atmospheric Chemistry and Physics, 2012, vol. 12, pp. 4935-4950.

5. Zhang Q.H., Zhang J.P. X ue H.W. The challenge of improving visibility in Beijing. Atmospheric Chemistry and Physics, 2010, vol. 10, pp. 7821-7827.

6. Singh A., Dey S. Influence of aerosol composition on visibility in megacity Delhi. Atmospheric Environment, 2012, vol. 62, pp. 367-373.

7. Denjean C., Formenti P., Picquet-V arrault B., K atrib Y., Pangui E., Zapf P., Doussin J.F. A new experimental approach to study the hydroscopic and optical properties of aerosol: application to ammonium sulfate particles. Atmospheric Measurement Techniques, 2014, vol. 7, pp. 183-197.

8. M ali R.R., V ashistha R.D. V isibility measurement technique and its application in aviation services at international airports in India, available at: https: www.wmo.int/pages/prog/www/IM OPpublications//OM -82-TEC0-2005/ Posters/P1(08)_India_2_Mali.pdf (accessed 1 September 2016).

9. Okey K., N wofor Ph.D. Seasonal levels of meteorological visibility at port-harcourt Nigeria and possible links to aerosol loading and humidification. The Pacific Journal of Science and Technology, 2010, vol. 11, no. 2, available at: http: //www.akamaiuniversity.us/PJST.htm (accessed 1 September 2016).

10. Ginoux P., Horowitz L.W., Ramaswamy V., Geogdzhayev I.V., Holben B.N., Stenchikov G., Tie X. Evaluation of aerosol distribution and optical depth in the geophysical fluid dynamics laboratory coupled model CM2.1 for present climate. Journal of Geophysical Research, 2006, vol. 111, D22210, pp. 1-21. DOI: 10.1029/2005J D006707

11. Chen J., Qiu Sh., Sjang J., Wilfrid M.F.O., Liu X., Tian H., Boman J. Impact of relative humidity and water soluble constituents of $\mathrm{PM}_{2,5}$ on visibility impairment in Beijing, China. Aerosol and Air Quality Research, 2014, vol. 14, pp. 260-268.

12. B eyersdorf A.J ., Ziemba L.D., Chen G., Corr C.A., Crawford J.H., Diskin G.S., Moore R.H., Thornhill K.L., Winstead E.L., A nderson B.E. The impacts of aerosol loading, composition and water uptake on aerosol extinction variability in the Baltimore - Washington, D.C. region. Atmospheric Chemistry and Physics, 2016, vol. 16, pp. 1003-1015.

13. Petters M .D., K reidenwais S.M . A single parameter representation of hydroscopic growth and cloud condensation nucleus activity. Atmospheric Chemistry and Physics, 2007, vol. 7, pp. 1961-1971. 
14. Ionel I., Nicolae D., Popescu F., Talianu C., B elegante J., A postol G. M easuring air pollutants in an international Romania airport with point and open path instruments. Romanian Journal of Physics, 2011, vol. 56, no. 3-4, pp. 507-519.

15. Daley P.S., Naugle D.F. Measurements and analysis of airport emissions. Journal of Air Pollution Control Association, 1979, vol. 29, pp. 113-116.

Получено 27.10.2016

\section{Об авторах}

Анодина Татьяна Григорьевна (Москва, Российская Федерация) - доктор технических наук, профессор, председатель Межгосударственного авиационного комитета $(19017$, г. Москва, ул. Большая Ордынка, 22/2/1, e-mail: mak@mak.ru).

Магеррамов Ибрагим Шаиг оглы (Баку, Азербайджанская Республика) - аспирант, Национальное аэрокосмическое агентство (AZ1106, Азербайджанская Республика, г. Баку, ул. С.С. Ахундова, 1, e-mail: imaharramov@ hotmail.com).

\section{About the authors}

Tat'iana G. Anodina (M oscow, Russian Federation) - Doctor of Technical Sciences, Professor, Chairman of Intergovernmental Aviation Committee (22/2/1, Bolshaya Ordynka st., M oscow, 119017, Russian Federation, e-mail: mak@mak.ru).

Ibragim S. Magerramov (A zerbaijan Republic, Baku) - Postgraduate Student, National Aerospace Agency (1, S.S. A khundov st., Baku, AZ1106, A zerbaijan Republic, e-mail: imaharramov@ hotmail.com). 